\title{
Lymphangiografie zur Behandlung eines postoperativen Lymphlecks
}

Kim PH et al. Lymphangiography with or without Embolization for the Treatment of Postoperative Chylous Ascites. Ann Vasc Surg 2020; 68: 351-360

Die chirurgische Behandlung eines Lymphlecks in Abdomen und Becken geht mit einer hohen Mortalität und Morbidität einher. Deshalb gewinnt zunehmend die Lymphadenografie (LAG) als minimalinvasive Therapie an Bedeutung. Da Lymphlecks nur selten vorkommen, gibt es allerdings kaum Untersuchungen dazu. Kim et al. konnten in ihrer retrospektiven Studie nun zeigen, dass die LAG eine sichere und effektive Behandlungsmethode darstellt.

Die Autoren haben für diese Studie nicht nur die Daten von 15 eigenen Patienten ausgewertet, sondern auch ein systematisches Review zu diesem Thema durchgeführt. Ihre Patienten waren durchschnittlich $61,9( \pm 10,5)$ Jahre alt. Bei allen trat das Lymphleck (1610 ml/Tag) nach einer radikalen Tumorresektion in Abdomen und/oder Becken mit oder ohne Lymphknotenresektion auf. Meist litten sie unter einem Ovarial- oder Prostatakarzinom. Zwischen der Tumoroperation und den lymphatischen Interventionen vergingen im Mittel 49 Tage.

Bei allen Patienten war die LAG technisch erfolgreich, d. h. das Kontrastmittel konnte ins Lymphsystem injiziert und der ange- zielte Lymphgang damit angefärbt werden. Bei 12 Patienten konnte mindestens ein Leck lokalisiert werden. Auch die anschließende Embolisation (bei 10 von 12 Patienten) war bei allen Patienten erfolgreich, d. h. der Lymphgang konnte vollständig okkludiert werden. Die übrigen 5 Patienten wurden nicht embolisiert, entweder weil in der LAG keine Leckstelle gefunden werden konnte oder weil diese an einer ungünstigen Stelle lag.

Klinisch erfolgreich (Lymphleck vollständig behoben) waren die Eingriffe bei $80 \%$ (LAG) bzw. $60 \%$ (Embolisation). Komplikationen traten in keinem Fall auf. Die primäre bzw. sekundäre klinische Erfolgsrate (vollständiges Verschwinden des Lecks ohne Notwendigkeit weiterer Interventionen bzw. klinischer Erfolg nach mehreren Eingriffen) lag bei 66,7\% bzw. 93,3\%.

Die Literaturrecherche ergab 13 retrospektive Studien mit insgesamt 280 Patienten (einschließlich der eigenen 15); davon erfüllten 87 Patienten die Einschlusskriterien (postoperatives Lymphleck in Abdomen oder Becken behandelt mit lymphatischen Interventionen). In dieser Patientenkohorte lag die technische Erfolgsrate der LAG bzw. der Embolisation bei 97,7\% bzw. 97,4\%, die klinische Erfolgsrate bei 68,1\% bzw. 70,3\%.
Auch hier traten keine größeren Komplikationen auf; kleinere Komplikationen wie Unterschenkelödeme oder periaortale Hämatome gab es bei 5,4\% (Embolisation) bzw. 1,2\% (LAG) der Patienten. Von den 30 Patienten, bei denen ein Eingriff nicht ausreichte, um das Lymphleck zu beheben, unterzogen sich 14 Patienten weiteren lymphatischen Interventionen (sekundäre klinische Erfolgsrate 78,2\%).

\section{FAZIT}

Kann das Lymphleck in der LAG dargestellt werden, sollte dieses, soweit möglich, embolisiert werden, empfehlen die Autoren. Die Embolisation erhöht die klinische Erfolgsrate, ohne zu größeren Komplikationen zu führen. Bei $20 \%$ der Patienten konnte das Lymphleck auch nach wiederholten Eingriffen nicht behoben werden. Um unnötige lymphatische Interventionen bei solchen Patienten zu vermeiden, sollten Risikofaktoren für ein Therapieversagen identifiziert werden, so die Autoren.

Stephanie Gräwert, Leipzig 